\title{
Malignancy risk of thyroid nodules with repeated "atypia of undetermined significance/follicular lesion of undetermined significance" diagnosis
}

\author{
Berrin Çetinarslan, Alev Selek, Emine Kıvrakoğlu, Zeynep Cantürk, IIhan Tarkun, Zeynep Akyay
}

Kocaeli University Faculty of Medicine, Department of Endocrinology and Metabolism

\section{OBJECTIVES}

"Atypia of undetermined significance/follicular lesion of undetermined significance" (AUS/FLUS) is a heterogeneous diagnostic thyroid fine needle aspiration biopsy (FNAB) category. AUS/FLUS is comprised of cases that cannot be definitively diagnosed as benign, suspicious for/consistent with neoplasm, suspicious for malignancy or malignant. The recommended management strategy for these cases is to perform a repeat FNAB after an appropriate time course. Although the estimated risk of malignancy for AUS/FLUS is reported $5-15 \%$, there is growing evidence in the literature that suggest higher incidence of malignancy for this category The purpose of this study is to estimate the malignancy risk of repeated AUS/FLUS diagnosis of thyroid fine needle aspiration biopsies.

\section{METHODS}

We report retrospective analyzes of 56 cases with repeated AUS/FLUS diagnosis among 5396 thyroid FNABs. The demographic data and histologic follow-up were evaluated in the study. Histologic outcome was categorized as benign, malignant or well-differentiated tumors of uncertain malignant potential (WDT-UMP).
5396 FNABs

280 initial AUS/FLUS diagnosis (5.2\%)

63 repeated AUS/FLUS diagnosis (22\% of initial AUS/FLUS diagnosis)

(7 patiens lost to follow-up)

56 patients with surgical outcome were recruited to the study

\begin{tabular}{lc}
\hline $\begin{array}{l}\text { Table.1 Surgical outcome following a repeated diagnosis of atypia of undetermined } \\
\text { significance/follicular lesion of undetermined significance in thyroid FNAB }\end{array}$ \\
\hline Outcome & No. (\%) of Cases \\
Benign & $28(50 \%)$ \\
Malignant & $23(41 \%)$ \\
$\quad$ Papillary Cancer & $21(95 \%)$ \\
$\quad$ Follicular Cancer & $2(5 \%)$ \\
Well-differentiated tumors of uncertain malignant potential & $5(9 \%)$ \\
\hline
\end{tabular}

\begin{tabular}{lcc}
\hline $\begin{array}{l}\text { Table.2 Surgical outcome of patients following a repeated diagnosis of atypia of undetermined } \\
\text { significance/follicular lesion of undetermined significance according to gender }\end{array}$ \\
\hline Outcome & \multicolumn{2}{c}{ No. (\%) of Cases } \\
& Female & Male \\
Benign & $26(56 \%)$ & $2(22 \%)$ \\
Malignant & $18(38 \%)$ & $5(56)$ \\
$\quad$ Papillary Cancer & $16(95 \%)$ & $5(100 \%)$ \\
$\quad$ Follicular Cancer & $2(5 \%)$ & $0(0 \%)$ \\
Well-differentiated tumors of uncertain malignant potential & $3(6 \%)$ & $2(22 \%)$
\end{tabular}

\section{RESULTS}

280 patients with AUS/FLUS diagnosis on initial FNAB were constituted $5.2 \%$ of all FNAB group, among them $63(22 \%)$ was diagnosed again AUS/FLUS. A total of 56 cases with repeated AUS/FLUS diagnosis and surgical outcome were recruited to the study (figure.1).

The study group included $47(84 \%)$ females and $9(16 \%)$ males with a mean age of $47.7( \pm 13)$ years. There was no difference between mean age in both groups, $48.4( \pm 13)$ for females, $44( \pm 12)$ for males $(p>0,05)$. The nodule size ranged from 0.9 to $6 \mathrm{~cm}($ mean, $1.8 \pm 1.0 \mathrm{~cm})$.

Among 56 cases with a repeated diagnosis of AUS/FLUS by FNAB, histologic follow-up revealed $28(50 \%)$ patients were benign, $23(41 \%)$ patients were malignant and $5(9 \%)$ patients were WDT-UMP. These results are summarized in Table 1 . Papillary thyroid carcinoma accounted for $95 \%$ of all malignant outcomes at surgical resection (beside WDT-UMP). The mean age of malignant and benign groups were similar in both groups, even in subgroup analysis according to gender. The results of the surgical outcomes according to gender revealed that, malignant outcome was higher in male patients although this difference was not statistically significant (56\% in males and $38 \%$ of females) (table 2$)$. If patients diagnosed with WDTUMP were analyzed in malignant group, the malignancy outcome increased to $78 \%$ in males while $44,5 \%$ in females $(p=0,07)$.

\section{CONCLUSIONS}

AUS/FLUS is a heterogeneous FNAB category. The thyroid nodules in this category cannot be characterized as benign due to nuclear or architectural atypia of the follicular cells. However, the atypia is also insufficient to meet the criteria for diagnosing suspicious for malignancy, malignant or follicular neoplasm categories [2]. According to BS an AUS/FLUS diagnosis is obtained in $3 \%$ to $6 \%$ of thyroid FNABs $[3,4]$. The higher rates of this diagnosis might indicate overuse of this category. The incidence of AUS/FLUS diagnosis in our cohort was $\% 5,2$ which was consistent with the suggested incidence rate of BS.

The management recommendation of AUS/FLUS category according to BS is repeating FNAB at an appropriate interval, as the anticipated risk of malignancy is low, $5-15 \%$ [2]. However, the majority of the recently published data demonstrate higher malignancy risk than anticipated in BS $[7,8]$. The data concerning the malignancy risk of repeated AUS/FLUS diagnosis is controversial. Paul et al have showed no statistically significant difference in malignancy rate among patients who went directly to surgery after a single AUS diagnosis and patients having 2 successive AUS FNAB diagnoses (41-43\% respectively) [7]. On the other hand, Wong et al reported the malignancy incidence of $25.6 \%$ in single AUS/FLUS diagnosis while $38.8 \%$ after repeated AUS/FLUS diagnosis [8]. The malignancy rate among 56 repeated AUS/FLUS diagnosis in our study was $41 \%$. This rate is three times higher than reported in BS but consistent with recent studies.

The evidence supporting higher malignancy rate of this category is increasing. Even the pathologic classification is not changed the clinical outcome data would be revised. The reported malignancy rate of follicular neoplasm or suspicious for a follicular neoplasm (FN/SFN) was $15 \%-30 \%$, for whom surgery is recommended management. Therefore, the management strategy of AUS/FLUS should also be revised as the malignancy rate was same or even higher than FN/SFN.

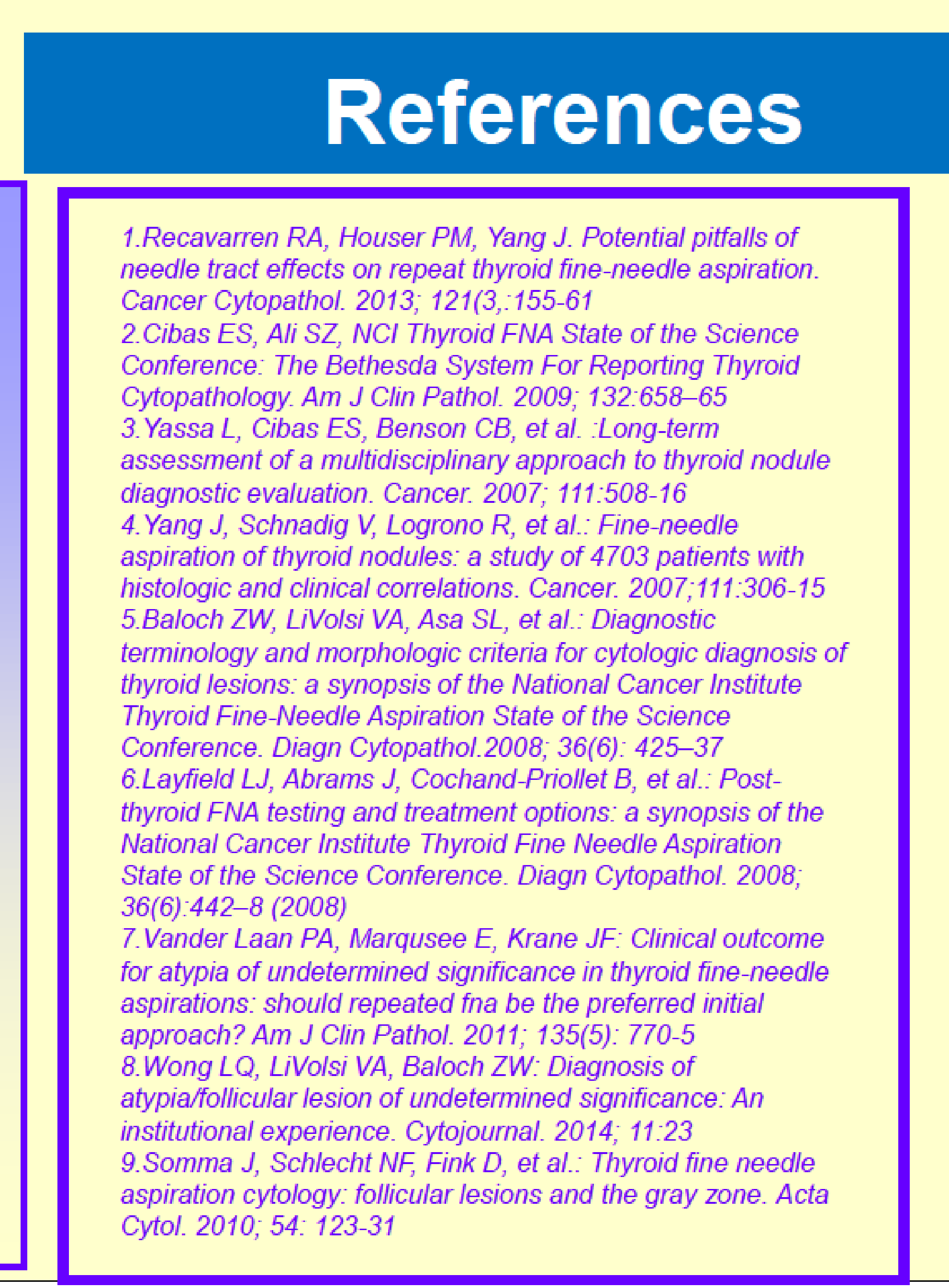

